\title{
Improvement of Chemistry Teaching Practice to Deep Learning
}

\author{
Li Qinghua \\ Nanchang Institute of Science and Technology, Nanchang, China
}

Keywords: deep learning; chemistry teaching; practice improvement

\begin{abstract}
Deep learning plays an important role in improving students' academic literacy and comprehensive ability. The deep learning is integrated in junior high school chemistry, which means that chemistry education is moving toward a higher level. With the continuous reform of the education system, China has paid more and more attention to education. As a foundation education for high school chemistry, junior high school chemistry should constantly explore and innovate teaching models, explore more perfect teaching strategies, and improve the professional level of teachers. This paper is based on the analysis of current junior school chemistry education, studies the improvements on chemistry teaching practice to deep learning.
\end{abstract}

\section{The Concept of Deep Learning}

The concept of deep learning originally originated from the research of foreign artificial neural networks. It is a learning structure with multiple perceptrons. The definition of deep learning was first mentioned in the article "Promoting Students' Deep Learning": Deep learning is a learning method to critically understanding and mastery of information and knowledge based on active learning, and finally build a systematic and effective knowledge system, and is also an effective way to develop discipline literacy and problem-solving abilities. In short, learners can actively and unsupervisedly conduct independent learning, think actively, connect knowledge with daily life, and solve problems in practice.

Different from shallow learning, the memory method emphasized by deep learning is not mechanized memory, but the memory and reflection on the basis of understanding, and the knowledge system is more regular and systematic, and can integrate the learning to solve practical problems. Deep learning is proactive, lifelong, integrated, and critical.

\section{Problems in Chemistry Teaching Practice}

\subsection{Students' lack of learning interest and initiative}

The learning of junior high school chemistry is the foundation for high school chemistry, which also means that the teaching contains the recitation of a lot of basic knowledge. For students who are new to this new discipline, the boring concepts, obscure explanations, and teachers' wrong teaching methods can all increase the difficulty of chemistry learning to some extent. Influenced by traditional teaching mode, although the "education reform" has been promoted to the present, it has not yet been implemented into practice. The improvement of the teaching model is only at the surface stage, and some older teachers are still banned in the "cramming" teaching method. The students who should have been the main body of the classroom are placed in the object position, and the teacher's "teaching" is emphasized too much and the students' "learning" is ignored. Students can only passively accept, but cannot perform "deep learning" to follow the teacher's ideas to sort out the theoretical knowledge of textbooks, and boring classroom teaching has greatly restricted the enthusiasm and initiative of students.

\subsection{Teachers' excessive relying on multimedia technology}

With the rapid development of Internet technology, the classroom model that uses information technology to carry out education and teaching has gradually been recognized by teachers and students, and has become the mainstream teaching method to replace traditional teaching methods. 
However, teachers are easy to take it as the main means of classroom teaching, while ignoring their own words and deeds, expanding teaching and in-depth exploration, and blindly expressing the teaching content through PPT and micro video. If the display material cannot be strictly selected, the too compact and intensive material display will not only disorganized, but also reduce the classroom teaching efficiency. While distracting students' attention, the fast-paced teaching method also makes students unable to think and understand in depth. The teacher's "teaching" and the students' "learning" are greatly reduced. It is also the excessive dependence on intelligent technology that leads to the deviation of the teaching direction of some junior middle school chemistry teachers, ignoring the cultivation of students' comprehensive quality and ability. Blindly transferring knowledge through multimedia, and lack of communication with students and necessary communication feedback will stifle students' interest in learning and impede the overall development of students.

\subsection{The lack of practical operation}

Chemistry, like physics, requires the assistance of practical courses to help a deeper understanding of theoretical knowledge. However, the imperfection of laboratory infrastructure and the replacement of experimental course by teacher's theoretical course will weaken the construction of the core knowledge system to a certain extent. Studies have shown that a 45-minute course, students concentrate on only about 15 minutes, and the use of chemical practice can effectively extend the 15 minutes, allowing students to focus more on the understanding of chemical theory, greatly improving teaching efficiency. The lack of practical operation makes it impossible to experience experiment personally. The practical result can only be obtained from the book. Not only can it not establish a clear image in the mind, but only the teaching of the teaching material knowledge is contrary to the original intention of the chemical practice discipline. Students cannot master the basic operational skills of chemical experiments and explore the format of thinking, and the ability to solve practical problems is low. The experimental exploration process is only the formality, and the teaching effect is greatly reduced.

\section{Benefits from Deep Learning of Chemistry Teaching Practice}

When students in the third grade first came into contact with chemistry, they were mostly overwhelmed and did not know how to learn. Or there is a situation where "I understand when I hear it, but I am wrong when I do it." In the final analysis, it is because they have not fully grasped the contents of the teacher's teaching. For junior high school students, their mental development is not yet mature, easy to be influenced by the things around them, so it is difficult to concentrate on the classroom. The concept is boring, the course is difficult, and the teacher can't integrate and inspire students to think independently, etc., which is the drawback of traditional chemistry teaching class. In the long run, the enthusiasm and thinking initiative of students are suppressed, the mood is low, and the classroom atmosphere is poor, making the chemical become the difficult discipline, which has become the biggest weakness of junior high school students. Deep learning in chemistry teaching practice solves the problem, and there are several benefits in the integration of deep learning in chemistry teaching practice.

First of all, it can help students form a positive learning attitude and correct values, and the teaching practice of deep learning abandons the rigid preaching of teachers in traditional education classroom. Students actively think, and there is positive interaction and the collision of thinking between teachers and students, which builds a harmonious and loving learning community to promote the construction of student value system. Students are fully engaged in learning and development, which forms a good atmosphere with a virtuous circle.

Secondly, deep learning helps to improve learning efficiency and cultivate students' independent quality, critical thinking, and exploration and creation. The learning method of students proposing questions and solving problems independently gets rid of the single teaching, which is of great significance to the cultivation of students' comprehensive abilities.

Finally, the teaching method of deep learning is conducive to teachers adjusting the teaching 
strategy, adjusting the teaching objectives and progress according to the actual situation of each student's learning, avoiding the empty goal, and reducing the "neglecting the development of backward students to achieve the development of superior students" due to the inconsistency between the teaching progress and the student's ability to accept.

\section{Specific Case of Deep Learning of Chemistry Teaching Practice -Taking Metal and Metal Material as an Example}

\subsection{To determine teaching objective}

There is an overall objective for deep learning in each unit, which is the primary condition for deep teaching. It is necessary to integrate isolated knowledge points of each chapter under the overall objective, explore the connections and differences, and closely integrate the content of the textbook with the reality of life, and promote the establishment of student systematic knowledge system and in-depth discussion of the content.

In the teaching of the Unit 8 of Metal and Metal Material, it is necessary to determine such teaching objectives. First, to understand the physical properties of metals and the chemical properties of common metals and to find out the laws, memorize the order of metal activity, master the method of preventing metal corrosion; secondly, to understand the metal minerals and important alloys common in life, and know which minerals can be extracted; and most importantly, to recognize the important role of metal and metal materials in social survival and development, and the huge impact of the indiscriminate disposal of discarded metal on the environment, so that the importance of metal sorting and recycling is known, and the protection and effective use of metal resources is understood.

\subsection{To design activity theme}

In view of the recognition of the importance of metal sorting processing and recycling, and protecting and effectively utilizing metal resources, the theme of the activity can be set to "Development Theme" and "Exploration Theme" respectively.

The Development Theme is to collect information on the utilization of resources and energy in China from the perspective of social development, and recognize the importance of effective use of resources and development of new energy, to learn how to identify the fuel that is least harmful to the environment when choosing energy, and to promote students to establish an environmental protection concept that cares for the environment and saves energy, and learns to live in harmony with the natural society.

The Exploration Theme is to explore the rational application of metal products according to the nature of the metal being studied, to analyze and select metal materials from the perspectives of chemistry and life, to achieve a perfect transition of metal and metal materials knowledge, and to practically combined the theory of teaching materials and life to help students establish a preliminary framework for analyzing problems.

\subsection{To determine activity form}

The form of activity cannot be constrained with experiment exploration according to deep learning. The exchanges, reports sharing, and processing between groups are also common forms of activity. In this unit, activities should be close to life, and students should start from the actual situation, find the common metal or alloy materials around them in groups, and evaluate them in terms of price, extension, hardness and gloss, and evaluate whether the metal or alloy finished product is scientifically sound or not. For instance, the most common cans are made of metal aluminum, and students can know why in the process of self-investigation. Because the aluminum oxide formed by the reaction of aluminum metal and oxygen can form a dense protective film, and the aluminum inside the can is prevented from continuing to chemically act. In addition, aluminum is not only easy to recycle as a low-cost metal material, also will not produce substances that pollute the environment. In this way, students not only have the concept of protecting the environment, but 
also deepen their understanding and memory of metal and metal materials in practice.

\subsection{To conduct objective evaluation}

This is the last and most important part of the deep learning teaching practice. The objective evaluation of the activity can feedback the information, compare with the target to find out the inadequacies of the activity, and find out improvement and commendation, give affirmation to students and provide theoretical preparations and lessons for the next activity. A scientific evaluation system can enhance students' subject quality and ability at key points.

\section{Challenges Faced by Teachers in Deep Learning}

Because the classroom for deep learning is self-exploration and learning by students, the classroom is open and difficult to control. In the classroom teaching practice of deep learning with students' in-depth discussion and critical thinking of accepting knowledge, and the problems raised by the students cannot be known in advance, it is inevitable that they will encounter problems difficult to answer. At this time, if the teacher's professional knowledge and ability are insufficient, the progress of the practice will be interrupted and delayed.

In addition, the uneven learning ability and situation of students will also reduce the quality of classroom practice. Some students in the group who have strong logical thinking skills and can quickly absorb knowledge, and some students have the weak learning ability. If teachers cannot find the problem in time to guide, it will violate the original intention of deep teaching, fail to reach the teaching objective and reduce the teaching effect.

\section{Suggestions on the Integration of Deep Learning in Chemistry Teaching Practice}

\subsection{To enhance teachers' discipline knowledge and teaching ability}

It is necessary for teachers to establish a philosophy of lifelong learning, and read a wide range of books and magazines on chemistry, and expand knowledge, read professional books to make up for the shortcomings in theory, and continue to learn to accumulate strategies, enhance their teaching ability, and enhance the theoretical foundation for practice and exploration.

Teachers are required to continuously innovate, practice curriculum reform policies, create an active and healthy classroom atmosphere, improve students' learning efficiency and quality, have an in-depth understanding of the textbook, take its essence to abandon its dross, predict the problems that students may propose in advance and think about strategies; set aside $20 \%$ of the time for classroom activities, in preparation for the class extension... and to create their own characteristic classroom.

\subsection{To establish a more scientific and reasonable teacher evaluation system}

It is undeniable that China's current compulsory education is still at the stage of exam-oriented education, and the final grades of students is the most important factor in measuring a teacher's professional knowledge and teaching ability. It is precisely because of this, the fierce competition in the industry and the tremendous pressure from all walks of life that have made teachers neglect the comprehensive quality of students. A scientific and reasonable evaluation system is conducive to reflecting efficiency and promoting fairness, so that teachers can concentrate more on the ability and comprehensive quality of students' self-learning, promote the healthy development of junior high school students, and truly implement the education reform in the new era.

\subsection{To implement individualized education and hierarchical teaching}

Compared with other disciplines, polarization is prone to occur in chemistry due to the first contact for junior high school students. Due to the uneven level of individuals, it is not possible to create a healthy learning atmosphere, which not only limits the development of students to a certain extent, but also is not conducive to the use of "deep learning" chemistry teaching. In individualized education, students can learn in accordance within the limits they can reach, which meets the 
different learning needs of students at different levels. The hierarchical teaching provides students with an environment and teaching conditions suitable for their further development and improvement so that each student can grow better.

\section{Conclusion}

Integrating "deep learning" into the improvement of chemistry teaching practice to improve the teaching power of junior high school chemistry and the learning level of students as a whole is a problem that needs to be solved urgently, and it is also the fundamental task of teachers. For chemistry, as the strange discipline for junior high school students, there are problems in both teaching and learning. The “deep learning” can improve students' logical thinking ability and self-learning ability, however, there still are some challenges in the implementation process. Teachers need to change their own way of thinking and cultivating strategies, start from the essence of chemistry, improve students' academic literacy and ability to solve practical problems, promote students' deep learning and exploration of chemistry, and give full play to the "deep learning".

\section{References}

[1] Hu Jiuhua, Luo Bin, Chen Ying. Improvement of Chemistry Teaching Practice to Deep Learning [J]. Curriculum Teaching Material and Method, 2017 (03).

[2] Zhang Faxin. The Connotation and Implementation Strategy of "Deep Learning" in Chemical Education [J]. Research of Modern Basic Education, 2015, 9 (19).

[3] Yan Naisheng. Classroom Situation under the Perspective of Deep Learning [J]. Research in Education Development, 2013 (12).

[4] Zhang Hao, Wu Xiujuan. The Connotation of Deep Learning and Basic Analysis on Cognitive Theory [J]. China Educational Technology, 2012, 10 (07).

[5] Liu Yan, Zhang Faxin. Practice and Exploration of the Promotion of Deep Learning in Chemistry Teaching [J]. Education in Chemistry, 2015 (9).

[6] Zhang Lijie. Problems and Improving Measures of Junior School Chemistry Teaching [J]. Learning Weekly, 2014 (08).

[7] Zhang Zhaoming. Exploration and Practice of Deep Learning in Junior School Chemistry Classroom [J]. A School Friend of English, 2017, 8 (07).

[8] Wang Peipei. Effective Exploration of Junior Chemistry Classroom Teaching in New Curriculum Standard [J]. Success, 2013 (01).

[9] Chen Jie. Teaching Design of Junior School Chemistry Based on Deep Learning -Taking Salt and Sucrose - The Solubility of Substance as an Example [J]. Popular Science, 2017 (09).

[10] Yu Wensen. The Teaching Significance and Cultivation of Key Competence [J]. Education Today, 2016 (03). 